Article

\title{
Representing Sums of Finite Products of Chebyshev Polynomials of Third and Fourth Kinds by Chebyshev Polynomials
}

\author{
Taekyun Kim ${ }^{1}$, Dae San Kim ${ }^{2}$, Dmitry V. Dolgy ${ }^{3}$ and Cheon Seoung Ryoo ${ }^{4, *}$
}

1 Department of Mathematics, Kwangwoon University, Seoul 139-701, Korea; tkkim@kw.ac.kr

2 Department of Mathematics, Sogang University, Seoul 121-742, Korea; dskim@sogang.ac.kr

3 Institute of Natural Sciences, Far Eastern Federal University, 690950 Vladivostok, Russia; d_dol@mail.ru

4 Department of Mathematics, Hannam University, Daejeon 306-791, Korea

* Correspondence: ryoocs@hnu.kr

Received: 05 June 2018; Accepted: 30 June 2018; Published: 3 July 2018

Abstract: Here, we consider the sums of finite products of Chebyshev polynomials of the third and fourth kinds. Then, we represent each of those sums of finite products as linear combinations of the four kinds of Chebyshev polynomials, which involve the hypergeometric function ${ }_{3} F_{2}$.

Keywords: Chebyshev polynomials; sums of finite products; hypergeometric function

MSC: 11B68; 33C45

\section{Introduction and Preliminaries}

We first recall here that, for any nonnegative integer $n$, the falling factorial polynomials $(x)_{n}$ and the rising factorial polynomials $\langle x\rangle_{n}$ are respectively given by:

$$
\begin{aligned}
&(x)_{n}=x(x-1) \cdots(x-n+1), \quad(n \geq 1),(x)_{0}=1, \\
&<x>_{n}=x(x+1) \cdots(x+n-1), \quad(n \geq 1), \quad<x>_{0}=1 .
\end{aligned}
$$

The two factorial polynomials are related by:

$$
(x)_{n}=(-1)^{n}<-x>_{n}, \quad<x>_{n}=(-1)^{n}(-x)_{n} .
$$

We will make use of the following.

$$
\frac{(2 n-2 s) !}{(n-s) !}=\frac{2^{2 n-2 s}(-1)^{s}<\frac{1}{2}>_{n}}{<\frac{1}{2}-n>_{s}}
$$

for any integers $n, s$ with $n \geq s \geq 0$.

$$
\begin{gathered}
B(x, y)=\int_{0}^{1} t^{x-1}(1-t)^{y-1} d t=\frac{\Gamma(x) \Gamma(y)}{\Gamma(x+y)}, \quad(\operatorname{Re}(x), \operatorname{Re}(y)>0), \\
\Gamma\left(n+\frac{1}{2}\right)=\frac{(2 n) ! \Gamma\left(\frac{1}{2}\right)}{2^{2 n} n !}, \quad(n \geq 0) .
\end{gathered}
$$

Here, $B(x, y)$ and $\Gamma(x)$ are respectively the Beta and Gamma functions. 
The hypergeometric function ${ }_{p} F_{q}\left(\begin{array}{l}a_{1}, \cdots, a_{p} \\ b_{1}, \ldots, b_{q}\end{array} ;\right)$ is defined by (see [1]):

$$
\begin{array}{r}
{ }_{p} F_{q}\left(\begin{array}{l}
a_{1}, \cdots, a_{p} \\
b_{1}, \cdots, b_{p}
\end{array} ; x\right)=\sum_{n=0}^{\infty} \frac{<a_{1}>_{n} \cdots<a_{p}>_{n}}{<b_{1}>_{n} \cdots<b_{q}>_{n}} \frac{x^{n}}{n !} \\
(p \leq q+1, \quad|x|<1) .
\end{array}
$$

In this paper, we will need only some basic knowledge about Chebyshev polynomials, which we recall here in below. The interested reader may want to refer to [1-3] for full accounts of this fascinating area of orthogonal polynomials.

The Chebyshev polynomials of the first, second, third and fourth kinds are respectively defined by the following generating functions.

$$
\begin{gathered}
\frac{1-x t}{1-2 x t+t^{2}}=\sum_{n=0}^{\infty} T_{n}(x) t^{n}, \\
\frac{1}{1-2 x t+t^{2}}=\sum_{n=0}^{\infty} U_{n}(x) t^{n}, \\
F(t, x)=\frac{1-t}{1-2 x t+t^{2}}=\sum_{n=0}^{\infty} V_{n}(x) t^{n}, \\
G(t, x)=\frac{1+t}{1-2 x t+t^{2}}=\sum_{n=0}^{\infty} W_{n}(x) t^{n} .
\end{gathered}
$$

One way of deriving their generating functions is from their trigonometric formulas. For example, those formulas for $V_{n}(x)$ and $W_{n}(x)$ are given by:

$$
\begin{aligned}
& V_{n}(\cos \theta)=\frac{\cos \left(n+\frac{1}{2}\right) \theta}{\cos \frac{\theta}{2}}, \\
& W_{n}(\cos \theta)=\frac{\sin \left(n+\frac{1}{2}\right) \theta}{\sin \frac{\theta}{2}} .
\end{aligned}
$$

They are explicitly expressed as in the following.

$$
\begin{aligned}
T_{n}(x)= & { }_{2} F_{1}\left(-n, n ; \frac{1}{2} ; \frac{1-x}{2}\right) \\
= & \frac{n}{2} \sum_{l=0}^{\left[\frac{n}{2}\right]}(-1)^{l} \frac{1}{n-l}\left(\begin{array}{c}
n-l \\
l
\end{array}\right)(2 x)^{n-2 l}, \quad(n \geq 1), \\
U_{n}(x) & =(n+1){ }_{2} F_{1}\left(-n, n+2 ; \frac{3}{2} ; \frac{1-x}{2}\right) \\
& =\sum_{l=0}^{\left[\frac{n}{2}\right]}(-1)^{l}\left(\begin{array}{c}
n-l \\
l
\end{array}\right)(2 x)^{n-2 l}, \quad(n \geq 0), \\
V_{n}(x) & ={ }_{2} F_{1}\left(-n, n+1 ; \frac{1}{2} ; \frac{1-x}{2}\right) \\
& =\sum_{l=0}^{n}\left(\begin{array}{c}
2 n-l \\
l
\end{array}\right) 2^{n-l}(x-1)^{n-l}, \quad(n \geq 0),
\end{aligned}
$$




$$
\begin{aligned}
W_{n}(x) & =(2 n+1){ }_{2} F_{1}\left(-n, n+1 ; \frac{3}{2} ; \frac{1-x}{2}\right) \\
& =(2 n+1) \sum_{l=0}^{n} \frac{2^{n-l}}{2 n-2 l+1}\left(\begin{array}{c}
2 n-l \\
l
\end{array}\right)(x-1)^{n-l}, \quad(n \geq 0) .
\end{aligned}
$$

The Chebyshev polynomials of the first, second, third and fourth kinds are also given by Rodrigues' formulas.

$$
\begin{gathered}
T_{n}(x)=\frac{(-1)^{n} 2^{n} n !}{(2 n) !}\left(1-x^{2}\right)^{\frac{1}{2}} \frac{d^{n}}{d x^{n}}\left(1-x^{2}\right)^{n-\frac{1}{2}}, \\
U_{n}(x)=\frac{(-1)^{n} 2^{n}(n+1) !}{(2 n+1) !}\left(1-x^{2}\right)^{-\frac{1}{2}} \frac{d^{n}}{d x^{n}}\left(1-x^{2}\right)^{n+\frac{1}{2}}, \\
(1-x)^{-\frac{1}{2}}(1+x)^{\frac{1}{2}} V_{n}(x)=\frac{(-1)^{n} 2^{n} n !}{(2 n) !} \frac{d^{n}}{d x^{n}}(1-x)^{n-\frac{1}{2}}(1+x)^{n+\frac{1}{2}}, \\
(1-x)^{\frac{1}{2}}(1+x)^{-\frac{1}{2}} W_{n}(x)=\frac{(-1)^{n} 2^{n} n !}{(2 n) !} \frac{d^{n}}{d x^{n}}(1-x)^{n+\frac{1}{2}}(1+x)^{n-\frac{1}{2}} .
\end{gathered}
$$

They have the following orthogonalities with respect to various weight functions.

$$
\begin{aligned}
\int_{-1}^{1}\left(1-x^{2}\right)^{-\frac{1}{2}} T_{n}(x) T_{m}(x) d x & =\frac{\pi}{\epsilon_{n}} \delta_{n, m}, \\
\int_{-1}^{1}\left(1-x^{2}\right)^{\frac{1}{2}} U_{n}(x) U_{m}(x) d x & =\frac{\pi}{2} \delta_{n, m}, \\
\int_{-1}^{1}\left(\frac{1+x}{1-x}\right)^{\frac{1}{2}} V_{n}(x) V_{m}(x) d x & =\pi \delta_{n, m}, \\
\int_{-1}^{1}\left(\frac{1-x}{1+x}\right)^{\frac{1}{2}} W_{n}(x) W_{m}(x) d x & =\pi \delta_{n, m},
\end{aligned}
$$

where:

$$
\epsilon_{n}=\left\{\begin{array}{ll}
1, & \text { if } n=0, \\
2, & \text { if } n \geq 1,
\end{array} \delta_{n}= \begin{cases}0, & \text { if } n \neq m \\
1, & \text { if } n=m\end{cases}\right.
$$

To proceed further, we let:

$$
\begin{array}{r}
\alpha_{n, r}(x)=\sum_{l=0}^{n} \sum_{i_{1}+i_{2}+\cdots+i_{r+1}=l}\left(\begin{array}{c}
r-1+n-l \\
r-1
\end{array}\right) V_{i_{1}}(x) V_{i_{2}}(x) \cdots V_{i_{r+1}}(x), \\
(n \geq 0, r \geq 1), \\
\beta_{n, r}(x)=\sum_{l=0}^{n} \sum_{i_{1}+i_{2}+\cdots+i_{r+1}=l}(-1)^{n-l}\left(\begin{array}{c}
r-1+n-l \\
r-1
\end{array}\right) W_{i_{1}}(x) W_{i_{2}}(x) \cdots W_{i_{r+1}}(x), \\
(n \geq 0, r \geq 1) .
\end{array}
$$

We note here that both $\alpha_{n, r}(x)$ and $\beta_{n, r}(x)$ are polynomials of degree $n$.

In the following, we assume that the polynomials with subscript $n$, like $p_{n}(x), q_{n}(x)$ and $r_{n}(x)$, have degree $n$.

The linearization problem in general consists of determining the coefficients $c_{n m}(k)$ in the expansion of the product of two polynomials $q_{n}(x)$ and $r_{m}(x)$ in terms of an arbitrary polynomial sequence $\left\{p_{k}(x)\right\}_{k \geq 0}$ :

$$
q_{n}(x) r_{m}(x)=\sum_{k=0}^{n+m} c_{n m}(k) p_{k}(x) .
$$

A special problem of this is the case when $p_{n}(x)=q_{n}(x)=r_{n}(x)$, which is called either the standard linearization or the Clebsch-Gordan-type problem. 
Another particular case is when $r_{m}(x)=1$, which is the so-called connection problem. If further $q_{n}(x)=x^{n}$, it is called the inversion problem for the sequence $\left\{p_{k}(x)\right\}_{k \geq 0}$.

In this paper, we will consider the sums of finite products of Chebyshev polynomials of the third and fourth kinds in (25) and (26). Then, we are going to express each of them as linear combinations of the four kinds of Chebyshev polynomials $T_{n}(x), U_{n}(x), V_{n}(x)$ and $W_{n}(x)$. Thus, our problem may be regarded as a generalization of the linearization problem. We obtain them by explicit computations and using Propositions 1 and Lemma 1. The general formulas in Proposition 1 can be derived by using orthogonalities and Rodrigues' formulas for Chebyshev polynomials and integration by parts.

Finally, we note that many problems in physics and engineering can be solved with the help of special functions; for instance, we let the reader refer to the excellent papers [4-6] in this direction.

The next two theorems are our main results in which the terminating hypergeometric functions ${ }_{3} F_{2}\left(\begin{array}{c}-n, a, b \\ d, e\end{array} ; 1\right)$ appear.

Theorem 1. Let $n, r$ be integers with $n \geq 0, r \geq 1$. Then we have following.

$$
\begin{aligned}
& \sum_{l=0}^{n} \sum_{i_{1}+i_{2}+\cdots+i_{r+1}=l}\left(\begin{array}{c}
r-1+n-l \\
r-1
\end{array}\right) V_{i_{1}}(x) V_{i_{2}}(x) \cdots V_{i_{r+1}}(x) \\
& =\frac{(-1)^{n}(2 n+2 r) !}{r ! 2^{2 r}\left(n+r-\frac{1}{2}\right)_{r}} \\
& \times \sum_{k=0}^{n} \frac{(-1)^{k} \epsilon_{k}}{(n-k) !(n+k) !} 3 F_{2}\left(\begin{array}{c}
k-n,-k-n, \frac{1}{2}-n-r \\
\frac{1}{2}-n,-2 n-2 r
\end{array} ; 1\right) T_{k}(x) \\
& =\frac{(-1)^{n}(2 n+2 r) !}{r ! 2^{2 r-2}\left(n+r-\frac{1}{2}\right)_{r-1}} \\
& \times \sum_{k=0}^{n} \frac{(-1)^{k}(k+1)}{(n-k) !(n+k+2) !} 3 F_{2}\left(\begin{array}{c}
k-n,-k-n-2, \frac{1}{2}-n-r \\
-\frac{1}{2}-n,-2 n-2 r
\end{array} ; 1\right) U_{k}(x) \\
& =\frac{(-1)^{n}(2 n+2 r) !}{r ! 2^{2 r}\left(n+r-\frac{1}{2}\right) r} \\
& \times \sum_{k=0}^{n} \frac{(-1)^{k}(2 k+1)}{(n-k) !(n+k+1) !}{ }^{3} F_{2}\left(\begin{array}{c}
k-n,-k-n-1, \frac{1}{2}-n-r \\
\frac{1}{2}-n,-2 n-2 r
\end{array} ; 1\right) V_{k}(x) \\
& =\frac{(-1)^{n}(2 n+2 r) !}{r ! 2^{2 r-1}\left(n+r-\frac{1}{2}\right)_{r-1}} \\
& \times \sum_{k=0}^{n} \frac{(-1)^{k}}{(n-k) !(n+k+1) !}{ }^{3} F_{2}\left(\begin{array}{c}
k-n,-k-n-1, \frac{1}{2}-n-r \\
-\frac{1}{2}-n,-2 n-2 r
\end{array} ; 1\right) W_{k}(x) .
\end{aligned}
$$

Theorem 2. Let $n, r$ be integers with $n \geq 0, r \geq 1$. Then we have following.

$$
\begin{aligned}
& \sum_{l=0}^{n} \sum_{i_{1}+i_{2}+\cdots+i_{r+1}=l}(-1)^{n-l}\left(\begin{array}{c}
r-1+n-l \\
r-1
\end{array}\right) W_{i_{1}}(x) W_{i_{2}}(x) \cdots W_{i_{r+1}}(x) \\
& =\frac{(-1)^{n}(2 n+2 r) !}{r ! 2^{2 r}\left(n+r+\frac{1}{2}\right)_{r}} \\
& \times \sum_{k=0}^{n} \frac{(-1)^{k} \epsilon_{k}}{(n-k) !(n+k) !} 3 F_{2}\left(\begin{array}{c}
k-n,-k-n,-\frac{1}{2}-n-r \\
\frac{1}{2}-n,-2 n-2 r
\end{array} ;\right) T_{k}(x)
\end{aligned}
$$




$$
\begin{aligned}
& =\frac{(-1)^{n}(2 n+1)(2 n+2 r) !}{r ! 2^{2 r-1}\left(n+r+\frac{1}{2}\right)_{r}} \\
& \times \sum_{k=0}^{n} \frac{(-1)^{k}(k+1)}{(n-k) !(n+k+2) !}{ }^{3} F_{2}\left(\begin{array}{c}
k-n,-k-n-2,-\frac{1}{2}-n-r \\
-\frac{1}{2}-n,-2 n-2 r
\end{array} 1\right) U_{k}(x) \\
& =\frac{(-1)^{n}(2 n+2 r) !}{r ! 2^{2 r}\left(n+r+\frac{1}{2}\right)_{r}} \\
& \times \sum_{k=0}^{n} \frac{(-1)^{k}(2 k+1)}{(n-k) !(n+k+1) !}{ }_{3} F_{2}\left(\begin{array}{c}
k-n,-k-n-1,-\frac{1}{2}-n-r \\
\frac{1}{2}-n,-2 n-2 r
\end{array}\right) V_{k}(x) \\
& =\frac{(-1)^{n}(2 n+1)(2 n+2 r) !}{r ! 2^{2 r}\left(n+r+\frac{1}{2}\right) r} \\
& \times \sum_{k=0}^{n} \frac{(-1)^{k}}{(n-k) !(n+k+1) !}{ }^{3} F_{2}\left(\begin{array}{c}
k-n,-k-n-1,-\frac{1}{2}-n-r \\
-\frac{1}{2}-n,-2 n-2 r
\end{array}\right) W_{k}(x) .
\end{aligned}
$$

As we know, the Bernoulli polynomials are not orthogonal polynomials, but Appell polynomials. In [7], the sums of finite products of Chebyshev polynomials in (25) and (26) were expressed as linear combinations of Bernoulli polynomials. Furthermore, the same has been done for the sums of finite products of Bernoulli, Euler and Genocchi polynomials in [8-10]. All of these were found by deriving Fourier series expansions for the functions closely connected with those various sums of finite products. For some other applications of Chebyshev polynomials, we let the reader refer to [11-13].

\section{Proof of Theorem 1}

Here, we will prove Theorem 1. For this purpose, we first state Proposition 1 and Lemma 1 that will be used in Sections 2 and 3.

The results in Proposition 1 can be derived by using the orthogonalities in (20)-(23) and the Rodrigues formulas in (16)-(19). The statements $(a)$ and $(b)$ in Proposition 1 are respectively from the Equations (23) and (35) of [14], while $(c)$ and $(d)$ are respectively from the Equations (22) and (37) of [15].

Proposition 1. Let $q(x) \in \mathbb{R}[x]$ be a polynomial of degree $n$. Then, we have the following.

$$
\begin{aligned}
& \text { (a) } q(x)=\sum_{k=0}^{n} c_{k, 1} T_{k}(x) \\
& \text { where } \quad c_{k, 1}=\frac{(-1)^{k} 2^{k} k ! \epsilon_{k}}{(2 k) ! \pi} \int_{-1}^{1} q(x) \frac{d^{k}}{d x^{k}}\left(1-x^{2}\right)^{k-\frac{1}{2}} d x,
\end{aligned}
$$

(b) $q(x)=\sum_{k=0}^{n} c_{k, 2} U_{k}(x)$

$$
\text { where } \quad c_{k, 2}=\frac{(-1)^{k} 2^{k+1}(k+1) !}{(2 k+1) ! \pi} \int_{-1}^{1} q(x) \frac{d^{k}}{d x^{k}}\left(1-x^{2}\right)^{k+\frac{1}{2}} d x,
$$

(c) $q(x)=\sum_{k=0}^{n} c_{k, 3} V_{k}(x)$

$$
\text { where } c_{k, 3}=\frac{(-1)^{k} 2^{k} k !}{(2 k) ! \pi} \int_{-1}^{1} q(x) \frac{d^{k}}{d x^{k}}(1-x)^{k-\frac{1}{2}}(1+x)^{k+\frac{1}{2}} d x,
$$




$$
\begin{aligned}
& \text { (d) } q(x)=\sum_{k=0}^{n} c_{k, 4} W_{k}(x) \\
& \text { where } c_{k, 4}=\frac{(-1)^{k} 2^{k} k !}{(2 k) ! \pi} \int_{-1}^{1} q(x) \frac{d^{k}}{d x^{k}}(1-x)^{k+\frac{1}{2}}(1+x)^{k-\frac{1}{2}} d x .
\end{aligned}
$$

Lemma 1. Let $l, m$ be nonnegative integers. Then, we have the following.

$$
\begin{aligned}
& \int_{-1}^{1}(1-x)^{m-\frac{1}{2}}(1+x)^{l-\frac{1}{2}} d x \\
& =\frac{2^{l+m}}{(l+m) !} \Gamma\left(l+\frac{1}{2}\right) \Gamma\left(m+\frac{1}{2}\right) \\
& =\frac{(2 l) !(2 m) ! \pi}{2^{l+m}(l+m) ! l ! m !} .
\end{aligned}
$$

Proof. By changing the variables $1+x=2 y$, the integral in (35) becomes:

$$
\begin{aligned}
2^{l+m} \int_{0}^{1} y^{l+\frac{1}{2}-1}(1-y)^{m+\frac{1}{2}-1} d y & =2^{l+m} \frac{\Gamma\left(l+\frac{1}{2}\right) \Gamma\left(m+\frac{1}{2}\right)}{\Gamma(l+m+1)} \\
& =\frac{2^{l+m}(2 l) ! \Gamma\left(\frac{1}{2}\right)(2 m) ! \Gamma\left(\frac{1}{2}\right)}{(l+m) ! 2^{2 l} l ! 2^{2 m} m !}
\end{aligned}
$$

where we used (5) and (6).

As was shown in [7], the following lemma can be obtained by differentiating Equation (10). It expresses the sums of finite products in (25) very neatly, which plays an important role in the following discussion.

Lemma 2. Let $n, r$ be integers with $n \geq 0, r \geq 1$. Then, we have the identity.

$$
\sum_{l=0}^{n} \sum_{i_{1}+i_{2}+\cdots+i_{r+1}=l}\left(\begin{array}{c}
r-1+n-l \\
r-1
\end{array}\right) V_{i_{1}}(x) \cdots V_{i_{r+1}}(x)=\frac{1}{2^{r} r !} V_{n+r}^{(r)}(x),
$$

where the inner sum runs over all nonnegative integers $i_{1}, i_{2}, \cdots, i_{r+1}$, with $i_{1}+i_{2}+\cdots+i_{r+1}=l$.

From (14), the $r$-th derivative of $V_{n}(x)$ is given by:

$$
V_{n}^{(r)}(x)=\sum_{l=0}^{n-r}\left(\begin{array}{c}
2 n-l \\
l
\end{array}\right) 2^{n-l}(n-l)_{r}(x-1)^{n-l-r} .
$$

In particular, we have:

$$
\begin{aligned}
& V_{n+r}^{(r+k)}(x)=\sum_{l=0}^{n-k}\left(\begin{array}{c}
2 n+2 r-l \\
l
\end{array}\right) 2^{n+r-l}(n+r-l)_{r+k}(x-1)^{n-k-l} . \\
& V_{n+r}^{(r+k)}(x)=\sum_{l=0}^{n-k}\left(\begin{array}{c}
2 n+2 r-l \\
l
\end{array}\right) 2^{n+r-l}(n+r-l)_{r+k}(x-1)^{n-k-l} .
\end{aligned}
$$

Here, we will show only (28) of Theorem 1, since (27), (29) and (30) can be proved similarly to (28). With $\alpha_{n, r}(x)$ as in (25), we let:

$$
\alpha_{n, r}(x)=\sum_{k=0}^{n} c_{k, 2} U_{k}(x)
$$


Then, from $(b)$ of Proposition 1, (36), (38) and integration by parts $k$ times, we have:

$$
\begin{aligned}
c_{k, 2}= & \frac{(-1)^{k} 2^{k+1}(k+1) !}{(2 k+1) ! \pi} \int_{-1}^{1} \alpha_{n, r}(x) \frac{d^{k}}{d x^{k}}\left(1-x^{2}\right)^{k+\frac{1}{2}} d x \\
= & \frac{(-1)^{k} 2^{k+1}(k+1) !}{(2 k+1) ! \pi 2^{r} r !} \int_{-1}^{1} V_{n+r}^{(r)}(x) \frac{d^{k}}{d x^{k}}\left(1-x^{2}\right)^{k+\frac{1}{2}} d x \\
= & \frac{2^{k+1}(k+1) !}{(2 k+1) ! \pi 2^{r} r !} \int_{-1}^{1} V_{n+r}^{(r+k)}(x)\left(1-x^{2}\right)^{k+\frac{1}{2}} d x \\
= & \frac{2^{k+1}(k+1) !}{(2 k+1) ! \pi 2^{r} r !} \sum_{l=0}^{n-k}(-1)^{n-k-l}\left(\begin{array}{c}
2 n+2 r-l \\
l
\end{array}\right) 2^{n+r-l} \\
& \quad \times(n+r-l)_{r+k} \int_{-1}^{1}(1-x)^{n-l+1-\frac{1}{2}}(1+x)^{k+1-\frac{1}{2}} d x .
\end{aligned}
$$

From (40), (35), we get:

$$
\begin{aligned}
c_{k, 2} & =\frac{2^{k+1}(k+1) !}{(2 k+1) ! \pi 2^{r} r !} \\
& \times \sum_{l=0}^{n-k} \frac{(-1)^{n-k-l}(2 n+2 r-l) ! 2^{n+r-l}(n+r-l) !(2 k+2) !(2 n-2 l+2) ! \pi}{l !(2 n+2 r-2 l) !(n-k-l) ! 2^{n-l+k+2}(n-l+k+2) !(n-l+1) !(k+1) !} \\
& =\frac{(-1)^{n-k}(k+1)}{r !} \\
& \times \sum_{l=0}^{n-k} \frac{(-1)^{l}(2 n+2 r-l) !(n+r-l) !(2 n+2-2 l) !}{l !(n-k-l) !(n+k-l+2) !(2 n+2 r-2 l) !(n+1-l) !} .
\end{aligned}
$$

Using (3) and (4), (41) is equal to:

$$
\begin{aligned}
c_{k, 2} & =\frac{(-1)^{n-k}(k+1)(2 n+2 r) !}{r !(n-k) !(n+k+2) !} \\
& \times \sum_{l=0}^{n-k} \frac{(-1)^{l}(n-k)_{l}(n+k+2)_{l}<\frac{1}{2}-n-r>_{l} 2^{2 n-2 l+2}(-1)^{l}<\frac{1}{2}>_{n+1}}{l !(2 n+2 r)_{l} 2^{2 n+2 r-2 l}(-1)^{l}<\frac{1}{2}>_{n+r}<\frac{1}{2}-n-1>_{l}} . \\
& =\frac{(-1)^{n}(2 n+2 r) !}{r ! 2^{2 r-2}\left(n+r-\frac{1}{2}\right)_{r-1}} \\
& \times \frac{(-1)^{k}(k+1)}{(n-k) !(n+k+2) !} \sum_{l=0}^{n-k} \frac{k-n>_{l}<-k-n-2>_{l}<\frac{1}{2}-n-r>_{l}}{<-\frac{1}{2}-n>_{l}<-2 n-2 r>_{l} l !} \\
& =\frac{(-1)^{n}(2 n+2 r) !}{r ! 2^{2 r-2}\left(n+r-\frac{1}{2}\right)_{r-1}} \\
& \times \frac{(-1)^{k}(k+1)}{(n-k) !(n+k+2) !} F_{2}\left(\begin{array}{c}
k-n,-k-n-2, \frac{1}{2}-n-r \\
-\frac{1}{2}-n,-2 n-2 r
\end{array}\right) .
\end{aligned}
$$

Now, the Equation (28) in Theorem 1 follows from (39) and (42).

\section{Proof of Theorem 2}

In this section, we will show (31) of Theorem 2, as (32)-(34) can be treated analogously to (31). The following lemma can be obtained by differentiating (11) and is stated as Lemma 3 in [7]. 
Lemma 3. Let $n, r$ be integers with $n \geq 0, r \geq 1$. Then, we have the following identity.

$$
\begin{aligned}
& \sum_{l=0}^{n} \sum_{i_{1}+i_{2}+\cdots+i_{r+1}=l}(-1)^{n-l}\left(\begin{array}{c}
r-1+n-l \\
r-1
\end{array}\right) W_{i_{1}}(x) W_{i_{2}}(x) \cdots W_{i_{r+1}}(x) \\
& =\frac{1}{2^{r} r !} W_{n+r}^{(r)}(x)
\end{aligned}
$$

where the inner sum runs over all nonnegative integers $i_{1}, i_{2}, \cdots, i_{r+1}$, with $i_{1}+i_{2}+\cdots+i_{r+1}=l$.

From (15), the $r$-th derivative of $W_{n}(x)$ is given by:

$$
W_{n}^{(r)}(x)=(2 n+1) \sum_{l=0}^{n-r} \frac{2^{n-l}}{2 n+1-2 l}\left(\begin{array}{c}
2 n-l \\
l
\end{array}\right)(n-l)_{r}(x-1)^{n-l-r} .
$$

In particular,

$$
\begin{aligned}
& W_{n+r}^{(r+k)}(x) \\
& =(2 n+1) \sum_{l=0}^{n-k} \frac{2^{n+r-l}}{2 n+2 r+1-2 l}\left(\begin{array}{c}
2 n+2 r-l \\
l
\end{array}\right)(n+r-l)_{r+k}(x-1)^{n-k-l} .
\end{aligned}
$$

Here, we will show only (31) of Theorem 2, since (32)-(34) can be proven analogously to (31). With $\beta_{n, r}(x)$ as in (26), we put:

$$
\beta_{n, r}(x)=\sum_{k=0}^{n} c_{k, 1} T_{k}(x)
$$

Then, from (a) of Proposition 1, (43), (45) and integration by parts $k$ times, we have:

$$
\begin{aligned}
c_{k, 1}= & \frac{(-1)^{k} 2^{k} k ! \epsilon_{k}}{(2 k) ! \pi} \int_{-1}^{1} \beta_{n, r}(x) \frac{d^{k}}{d x^{k}}\left(1-x^{2}\right)^{k-\frac{1}{2}} d x \\
= & \frac{(-1)^{k} 2^{k} k ! \epsilon_{k}}{(2 k) ! \pi 2^{r} r !} \int_{-1}^{1} W_{n+r}^{(r)}(x) \frac{d^{k}}{d x^{k}}\left(1-x^{2}\right)^{k-\frac{1}{2}} d x \\
= & \frac{2^{k} k ! \epsilon_{k}}{(2 k) ! \pi 2^{r} r !} \int_{-1}^{1} W_{n+r}^{(r+k)}(x)\left(1-x^{2}\right)^{k-\frac{1}{2}} d x \\
= & \frac{(2 n+1) 2^{k} k ! \epsilon_{k}}{(2 k) ! \pi 2^{r} r !} \sum_{l=0}^{n-k} \frac{(-1)^{n-k-l} 2^{n+r-l}}{2 n+2 r+1-2 l}\left(\begin{array}{c}
2 n+2 r-l \\
l
\end{array}\right) \\
& \quad \times(n+r-l)_{r+k} \int_{-1}^{1}(1-x)^{n-l-\frac{1}{2}}(1+x)^{k-\frac{1}{2}} d x .
\end{aligned}
$$

From (47), (35) and after some simplifications, we get:

$$
\begin{aligned}
c_{k, 2} & =\frac{(2 n+1) \epsilon_{k}(-1)^{n-k}}{r !} \\
& \times \sum_{l=0}^{n-k} \frac{(-1)^{l}(2 n+2 r-l) !(n+r-l) !}{l !(n-k-l) !(n+k-l) !(2 n+2 r-2 l+1) !(n-l) !} \\
& =\frac{2(2 n+1) \epsilon_{k}(-1)^{n-k}}{r !} \\
& \times \sum_{l=0}^{n-k} \frac{(-1)^{l}(2 n+2 r-l) !(n+r-l+1) !(2 n-2 l) !}{l !(n-k-l) !(n+k-l) !(2 n+2 r-2 l+2) !(n-l) !} .
\end{aligned}
$$


Using (3) and (4), (48) is equal to:

$$
\begin{aligned}
c_{k, 1}= & \frac{2(2 n+1)(2 n+2 r) ! \epsilon_{k}(-1)^{n-k}}{r !(n-k) !(n+k) !} \\
\times & \sum_{l=0}^{n-k} \frac{(-1)^{l}(n-k)_{l}(n+k)_{l}<\frac{1}{2}-n-r-1>_{l} 2^{2 n-2 l}(-1)^{l}<\frac{1}{2}>_{n}}{l !(2 n+2 r)_{l} 2^{2 n+2 r+2-2 l}(-1)^{l}<\frac{1}{2}>_{n+r+1}<\frac{1}{2}-n>_{l}} \\
= & \frac{(2 n+1)(-1)^{n}(2 n+2 r) !}{r ! 2^{2 r+1}\left(n+r+\frac{1}{2}\right)_{r+1}} \\
\times & \frac{(-1)^{k} \epsilon_{k}}{(n-k) !(n+k) !} \sum_{l=0}^{k} \frac{<k-n>_{l}<-k-n>_{l}<-\frac{1}{2}-n-r>_{l}}{<\frac{1}{2}-n>_{l}<-2 n-2 r>_{l}} \\
& =\frac{(-1)^{n}(2 n+2 r) !}{r ! 2^{2 r}\left(n+r+\frac{1}{2}\right)_{r}} \\
& \times \frac{(-1)^{k} \epsilon_{k}}{(n-k) !(n+k) !}{ }^{3} F_{2}\left(\begin{array}{c}
k-n,-k-n,-\frac{1}{2}-n-r \\
\frac{1}{2}-n,-2 n-2 r
\end{array}\right) .
\end{aligned}
$$

Now, Equation (31) in Theorem 2 follows from (46) and (49).

Remark 1. As we noted earlier, Lemmas 2 and 3 play crucial roles and express sums of finite products in (25) and (26) very neatly as higher-order derivatives of $V_{n}(x)$ and $W_{n}(x)$. These could be derived by noting that Chebyshev polynomials are special cases of Jacobi polynomials and using the general formula for the derivative of Jacobi polynomials. Indeed, their Jacobi polynomial expressions and the derivatives of the Jacobi polynomials are as follows:

$$
\begin{aligned}
& V_{n}(x)=P_{n}^{(-1 / 2,1 / 2)}(x) / P_{n}^{(-1 / 2,1 / 2)}(1), \\
& W_{n}(x)=P_{n}^{(1 / 2,-1 / 2)}(x) / P_{n}^{(1 / 2,-1 / 2)}(1), \\
& \frac{d}{d x} P_{n}^{(a, b)}(x)=\frac{1}{2}(n+a+b+1) P_{n-1}^{(a+1, b+1)}(x) .
\end{aligned}
$$

\section{Conclusions}

The linearization problem in general consists of determining the coefficients $c_{n m}(k)$ in the expansion of the product of two polynomials $q_{n}(x)$ and $r_{m}(x)$ in terms of an arbitrary polynomial sequence $\left\{p_{k}(x)\right\}_{k \geq 0}$ :

$$
q_{n}(x) r_{m}(x)=\sum_{k=0}^{n+m} c_{n m}(k) p_{k}(x)
$$

Along this line and as a generalization of this, we considered sums of finite products of Chebyshev polynomials of the third and fourth kinds and represented each of those sums of finite products as linear combinations of the four kinds of Chebyshev polynomials, which involve the hypergeometric function ${ }_{3} F_{2}$. It is certainly possible to represent such sums of finite products by other orthogonal polynomials, which is our ongoing project.

Author Contributions: T.K. and D.S.K. conceived the framework and structured the whole paper; T.K. wrote the paper; C.S.R. and D.D.V. checked the results of the paper; D.S.K. and C.S.R. completed the revision of the article.

Funding: This work was supported by the National Research Foundation of Korea (NRF) grant funded by the Korean government (MEST) (No. 2017R1A2B4006092)

Acknowledgments: The authors would like to thank the referees for their valuable comments, which improved the original manuscript in its present form.

Conflicts of Interest: The authors declare no conflict of interest. 


\section{References}

1. Andrews, G.E.; Askey, R.; Roy, R. Special Functions; Encyclopedia of Mathematics and Its Applications 71; Cambridge University Press: Cambridge, UK, 1999.

2. Beals, R.; Wong, R. Special Functions and Orthogonal Polynomials; Cambridge Studies in Advanced Mathematics 153; Cambridge University Press: Cambridge, UK, 2016.

3. Wang, Z.X.; Guo, D.R. Special Functions; Guo, D.R., Xia, X.J., Trans.; World Scientific Publishing Co., Inc.: Teaneck, NJ, USA, 1989.

4. Marin, M. A temporally evolutionary equation in elasticity of micropolar bodies with voids. Politehn. Univ. Buchar. Sci. Bull. Ser. A Appl. Math. Phys. 1998, 60, 3-12.

5. Marin, M.; Stan, G. Weak solutions in elasticity of dipolar bodies with stretch. Carpath. J. Math. 2013, 29, 33-40.

6. Marin, D.; Baleanu, D. On vibrations in thermoelasticity without energy dissipation for micropolar bodies. Bound. Value Probl. 2016, 2016, 111. [CrossRef]

7. Kim, T.; Kim, D.S.; Dolgy, D.V.; Kwon, J. Representing Sums of finite products of Chebyshev polynomials of the third and fourth kinds by Chebyshev Polynomials. Preprints 2018, 2018060079. [CrossRef]

8. Agarwal, R.P.; Kim, D.S.; Kim, T.; Kwon, J. Sums of finite products of Bernoulli functions. Adv. Differ. Equ. 2017, 2017, 15. [CrossRef]

9. Kim, T.; Kim, D.S.; Jang, G.-W.; Kwon, J. Sums of finite products of Euler functions. In Advances in Real and Complex Analysis with Applications, Trends in Mathematics; Springer: New York, NY, USA, 2017; pp. $243-260$.

10. Kim, T.; Kim, D.S.; Jang, L.C.; Jang, G.-W. Sums of finite products of Genocchi functions. Adv. Differ. Equ. 2017, 2017, 17. [CrossRef]

11. Doha, E.H.; Abd-Elhameed, W.M.; Alsuyuti, M.M. On using third and fourth kinds Chebyshev polynomials for solving the integrated forms of high odd-order linear boundary value problems. J. Egyptian Math. Soc. 2015, 23, 397-405. [CrossRef]

12. Kruchinin, D.V.; Kruchinin, V.V. Application of a composition of generating functions for obtaining explicit formulas of polynomials. J. Math. Anal. Appl. 2013, 404, 161-171. [CrossRef]

13. Mason, J.C. Chebyshev polynomials of the second, third and fourth kinds in approximation, indefinite integration, and integral transforms. J. Comput. Appl. Math. 1993, 49, 169-178. [CrossRef]

14. Kim, D.S.; Kim, T.; Lee, S.-H. Some identities for Berounlli polynomials involving Chebyshev polynomials. J. Comput. Anal. Appl. 2014, 16, 172-180.

15. Kim, D.S.; Dolgy, D.V.; Kim, T.; Rim, S.-H. Identities involving Bernoulli and Euler polynomials arising from Chebyshev polynomials. Proc. Jangjeon Math. Soc. 2012, 15, 361-370. 